\title{
Schau mich an: Ich bin voll im Leben
}

\author{
Ursula Steiner-König \\ Dr. med., FMH-Delegierte ins Zentralkomitee "Tag der Kranken»
}

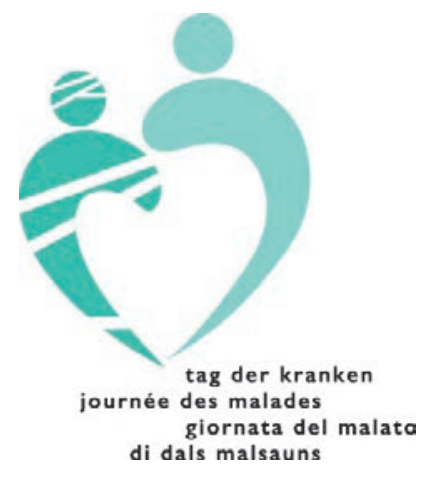

\author{
Nicht immer ist eine Krankheit oder eine Beeinträchtigung auf Anhieb erkennbar. \\ Betroffene wünschen sich meist, aktiv am Leben teilzuhaben und auch «voll im \\ Leben zu sein» - so lautet das Motto des diesjährigen «Tag der Kranken».
}

«... ich bin voll im Leben»: Diesen Eindruck haben wir doch gelegentlich auch von Patientinnen oder Patienten. Ihnen ist der diesjährige "Tag der Kranken» gewidmet: Menschen, die trotz Beeinträchtigung oder chronischer Krankheit aktiv am Leben teilnehmen und sich engagieren. Oft ist die Krankheit auf den ersten Blick gar nicht erkennbar. Gerade weil das Leiden nicht immer offensichtlich ist, ist es zentral - sei es in der medizinischen Behandlung als auch in der menschlichen Begegnung überhaupt -, die persönlichen und sozialen Ressourcen dieser Menschen zu berücksichtigen. Ganz besonders gilt das auch in der Arbeitswelt.

\section{Gäste im Leben unserer Patienten zu sein bedeutet, dass wir weder dauernde} Anwesenheit in deren Leben beanspruchen dürfen - noch müssen.

Im Nationalen Gesundheitsbericht 2015 finden wir folgendes Zitat:

«Manche meinen, Arzt und Patient sollten Partner sein. Ich sehe das anders. Meiner Ansicht nach sind wir Ärzte nicht Partner unserer Patienten. Wir sind Gäste im Leben unserer Patienten. Wir sind nicht Gastgeber.

"Servir et disparaître» - mit diesen Worten hat sich Dr. med. Ursula Steiner-König, Fachärztin für Psychiatrie und Psychotherapie, im Jahr 2006 aus dem Zentralvorstand der FMH verabschiedet. Schön, dass sie es mit dem «disparaître» nicht allzu ernst genommen hat! Denn danach engagierte sie sich noch über zehn Jahre als FMH-Delegierte im Zentralkomitee des "Tag der Kranken». Als Vizepräsidentin hat sie innerhalb des Komitees tatkräftig dazu beigetragen, die Beziehungen zwischen Gesunden und Kranken zu stärken und Verständnis für die Bedürfnisse der Kranken zu mehren. Nun übergibt Ursula Steiner ihr Mandat an Dr. med. Hans Kurt. Wir danken Ursula Steiner-König herzlich für ihr Engagement! Wie auch für ihre feinfühligen und berührenden Artikel, in denen stets auch eine Prise Humor Platz gefunden hat.

Und wir begrüssen ebenso herzlich Dr. med. Hans Kurt. Neu vertritt er die FMH im Zentralkomitee des «Tag der Kranken». Hans Kurt ist seit über 25 Jahren als Facharzt für Psychiatrie und Psychotherapie in einer interdisziplinären Gruppenpraxis in Solothurn tätig. Er kennt sich bestens aus in der Verbands- und Gesundheitspolitik, wo er verschiedene Mandate innehat. Wir wünschen Hans Kurt viel Freude an der neuen Aufgabe.
Wir sind nicht Priester in einer Kathedrale der Technologie» [1].

Gäste im Leben unserer Patienten zu sein bedeutet, dass wir weder dauernde Anwesenheit in deren Leben beanspruchen dürfen - noch müssen. Aber solange wir Gäste sind, konzentrieren wir uns voll und ganz auf den Gastgeber oder die Gastgeberin, beobachten aufmerksam und versetzen uns so gut wie möglich in deren Lebenssituation. Was haben wir schon von ihnen gehört? Was mit ihnen erlebt? Wir sollen uns erinnern, was uns dabei durch den Kopf gegangen ist, was wir empfunden haben: Diese Ausgangslage verdeutlicht, dass die Gastgebenden meist mehrerer Gäste bedürfen, um ihr Ziel, «voll im Leben zu sein», auf adäquate Weise umsetzen zu können.

\section{Was können wir Ärztinnen und Ärzte beitragen?}

Aufgrund meiner Erfahrungen als Psychiaterin und Psychotherapeutin denke ich zunächst an die Begleitung von Traumapatienten und deren Angehörigen. Bei Trauma denke ich vorwiegend an Menschen, denen etwas Unerwartetes, ihr bisheriges Leben in Frage Stellendes passiert ist: Sei es etwa ein schwerer Unfall, eine HIV- oder eine Krebserkrankung, eine Hirnblutung, ein Hörsturz, eine unvorhergesehene Entlassung am Arbeitsplatz, um nur einige Beispiele zu nennen. Wenn diese Menschen unsere Hilfe wünschen, bedeutet dies für uns Ärztinnen und Ärzte - abgesehen von der medizinisch indizierten Behandlung - ein genaues Hinsehen und Abklären, welche Ressourcen bei dieser Person vorhanden sind. Es ist unser Ziel, diese Menschen möglichst optimal auf ihren künftigen Lebensweg zu begleiten, ihnen aus dem Einbruch in ihrem Dasein hinauszuhelfen. Gelingt es uns, diese Menschen mit Geduld und Einfühlungsvermögen durch diese schwierige Anfangsphase zu begleiten? Getragen von 
der Einstellung, dass der Tunnel ein Ende haben kann, ermöglicht dies auch der betroffenen Person, wieder Licht zu erblicken. Angehörige benötigen eine solch geduldige Begleitung übrigens nicht minder. Viele Faktoren spielen dabei mit, und immer sollten wir uns unserer Rolle als Gast bewusst sein. Sie impliziert den nötigen Abstand zur Situation der Betroffenen.

\section{Voll im Leben - ermutigende Beispiele}

In einem Zeitungsartikel bin ich auf die Stiftung «Claire und George» [2] mit Sitz in Bern gestossen. Sie organisiert Hotelspitex-Dienstleistungen für Menschen mit Behinderungen. Die Stiftung versteht sich als «Drehscheibe, Kompetenzzentrum und Vermittlungsplattform» und sucht barrierefreie Zugangs- und Erholungswege zu vermitteln und anzuregen. Sie ermöglicht damit Menschen mit Behinderungen oder Beeinträchtigungen, Ferien zu geniessen - eben voll im Leben zu stehen.

Ein weiteres ermutigendes Beispiel ist «Kinderkrebs Schweiz» [3]. Die Organisation ist bestrebt, «Survivership Passports» aufzubauen, die erfolgreich Überlebenden den Zugang zu allen nötigen Informationen bezüglich ihrer durchgemachten Erkrankung öffnen soll. Eine Studie von 2014 hatte gezeigt, dass viele Survivors häufig trotz intensiver Begleitung nur über ungenaues Wissen zu ihrer Erkrankung verfügten. Es ist beachtlich, wie viele private Initiativen vorhanden sind und mithelfen, Menschen mit Beeinträchtigungen am vollen Leben teilhaben zu lassen.

\section{Medical Humanities - sich als Mensch verstanden fühlen}

In unseren Tagen ist kaum mehr von ärztlicher Kunst die Rede. Vielmehr begegnete ich in der Schweizerischen Ärztezeitung dem Begriff E-Nudging [4], wobei es darum geht, "für chronisch kranke Menschen die richtigen Anreize für eine gesündere Lebensweise zu schaffen». «To nudge» sei gleichbedeutend mit «jemanden leicht anstubsen" und kommt offenbar aus der Verhaltensökonomie. Die grosse Herausforderung bestehe darin, herauszufinden, «was für wen, unter welchen Umständen und für wie lange funktioniert» [4]. Ich muss gestehen, dass es mir dabei kalt den Rücken hinunterläuft. Es geht nicht mehr um das ganzheitliche Erfassen eines Leidens, sondern um dasjenige der gestörten körperlichen Funktion. Fühlt sich aber der Patient dabei verstanden, angenommen? Wie oft begegnen uns doch Menschen, die von Praxis zu Praxis wechseln weil sie sich eben nicht verstanden erleben. Einer Spanisch sprechenden Allgemeinmedizinerin passierte es kürzlich, dass eine südamerikanische Patientin ihr am Schluss des Erstgesprächs völlig unerwartet um den Hals fiel ... Was mochte das bedeutet haben: «Endlich eine Ärztin, die mich ernst nimmt, die mich versteht»? Jedenfalls kam da etwas zum Ausdruck, das über alles technische Wissen der Kollegin hinausging.

Die Philosophie spricht vom Suchen nach dem Ganzen in der Medizin. Jean Martin hat sich im Artikel «Quelle vision il y a 50 ans des enjeux éthiques en médecine?» [5] Gedanken gemacht und zitiert darin E. F. Torrey, einen amerikanischen Psychiater und Leiter eines Forschungszentrums. Dieser sagte in den 1970er Jahren, die technologische Revolution sei "mindless» und neutral, weil reine Wissenschaft einzig Wunsch nach Wissen sei und deren Neuerungen an sich weder gut noch böse seien. In der Verantwortung der Ärzteschaft stehe eine genügend ausführliche Information zu Handen der Gesellschaft; erst damit werde eine sinnvolle, aufgeklärte Meinungsbildung und Grenzsetzung möglich. Keinesfalls dürfe es zu einer Vogel-Strauss-Politik infolge mangelnden sozialen Engagements seitens der

\section{Der Ruf nach stärkerer Humanisierung der Medizin wird vernehmbar.}

Ärzteschaft kommen. Es dürfe nicht nur um Standespolitik und Tarife gehen. Zu Recht fragt sich Jean Martin, ob wir heute so viel weitergekommen sind. Auch Lazare Benaroyo weist in einem Artikel [6] darauf hin, dass seit den 1980er Jahren Ärzte zunehmend darüber enttäuscht sind, dass die ärztliche Kunst von der technischen Kompetenz in der Medizin verdrängt werde. Der Ruf nach stärkerer Humanisierung der Medizin wird vernehmbar. Der Autor spricht vom Paradox «Le soin doit précéder l'acte technique, alors que c'est l'acte technique qui guide maintenant le soin». Jede noch so ausgeklügelte Technologie stosse an eine Grenze: Diese Grenze, das ist der andere. Eben: Wir sind Gäste im Leben unserer Patienten.

Weitere Informationen zum Thema finden sich auf der Website des «Tag der Kranken»: www.tagderkranken.ch

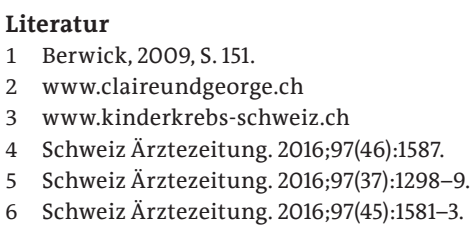

